\title{
Cued Spatial Attention Drives Functionally Relevant Modulation of the Mu Rhythm in Primary Somatosensory Cortex
}

\author{
Stephanie R. Jones, ${ }^{1 \star}$ Catherine E. Kerr, ${ }^{2 \star}$ Qian Wan, ${ }^{2,3}$ Dominique L. Pritchett, ${ }^{3}$ Matti Hämäläinen, ${ }^{1}$ \\ and Christopher I. Moore ${ }^{3}$ \\ ${ }^{1}$ Athinoula A. Martinos Center for Biomedical Imaging, Massachusetts General Hospital, Charlestown, Massachusetts 02129, ${ }^{2} \mathrm{Harvard}$ Osher Research \\ Center, Harvard Medical School, Boston, Massachusetts 02215, and ${ }^{3}$ McGovern Institute for Brain Research, Massachusetts Institute of Technology, \\ Cambridge, Massachusetts 02139
}

Cued spatial attention modulates functionally relevant alpha rhythms in visual cortices in humans. Here, we present evidence for analogous phenomena in primary somatosensory neocortex (SI). Using magnetoencephalography, we measured changes in the SI mu rhythm containing mu-alpha $(7-14 \mathrm{~Hz})$ and mu-beta $(15-29 \mathrm{~Hz})$ components. We found that cued attention impacted mu-alpha in the somatopically localized hand representation in SI, showing decreased power after attention was cued to the hand and increased power after attention was cued to the foot, with significant differences observed 500-1100 ms after cue. Mu-beta showed differences in a time window $800-850 \mathrm{~ms}$ after cue. The visual cue also drove an early evoked response beginning $\sim 70 \mathrm{~ms}$ after cue with distinct peaks modulated with cued attention. Distinct components of the tactile stimulus-evoked response were also modulated with cued attention. Analysis of a second dataset showed that, on a trial-by-trial basis, tactile detection probabilities decreased linearly with prestimulus mu-alpha and mu-beta power. These results support the growing consensus that cue-induced alpha modulation is a functionally relevant sensory gating mechanism deployed by attention. Further, while cued attention had a weaker effect on the allocation of mu-beta, oscillations in this band also predicted tactile detection.

\section{Introduction}

The historical view of electroencephalography/magnetoencephalography (EEG/MEG)-measured alpha rhythms $(7-14 \mathrm{~Hz})$ as a "resting" brain state is being challenged by evidence that they are actively and topographically deployed to gate information processing. Cued spatial attention leads to decreased alpha amplitudes in parietal-occipital EEG sensors contralateral to the attended site in visual (Worden et al., 2000; Kelly et al., 2006, 2009; Thut et al., 2006) and intersensory visual-auditory (Foxe et al., 1998; Fu et al., 2001) tasks. Alpha decreases are accompanied by increases in opposing hemifields, and lateralized alpha amplitudes predict reaction times and visual discriminability (Worden et al., 2000; Kelly et al., 2006, 2009; Thut et al., 2006). Recent work has also shown that attentional biases are tied to changes in com-

\footnotetext{
Received June 10, 2010; revised Aug. 2, 2010; accepted Aug. 12, 2010.

This work was supported by the National Institutes of Health (P41RR14075, K25MH072941, K01AT003459, 1R01-NS045130-01, and T32 GM007484), the National Science Foundation (0316933), and the 0sher Institute. We thank the anonymous reviewers for particularly thoughtful comments on earlier drafts of this manuscript and the Athinoula A. Martinos Center for Biomedical Imaging and the McGovern Institute for Brain Research for their support of this research.

*S.R.J. and C.E.K. contributed equally to this work.

Correspondence should be addressed to Stephanie R. Jones, Athinoula A. Martinos Center for Biomedical Imaging, Massachusetts General Hospital, 149 13th Street, Suite 2301, Charlestown, MA 02129. E-mail: srjones@ nmr.mgh.harvard.edu.

DOI:10.1523/JNEUROSCI.2969-10.2010

Copyright $\odot 2010$ the authors $\quad 0270-6474 / 10 / 3013760-06 \$ 15.00 / 0$
}

ponents of the broadband cue-induced evoked response (ER) in early visual cortices (Kelly et al., 2009).

In the somatosensory neocortex in humans, a spontaneous mu rhythm containing a complex of mu-alpha $(7-14 \mathrm{~Hz})$ and mu-beta (15-29 Hz) components is commonly observed above rolandic cortex (Tiihonen et al., 1989; Jones et al., 2009). These rhythms show an event-related desynchronization (ERD) with stimulation or movement, and in premovement periods, with a subsequent synchronization (ERS). There is an increase in alphaband ERS over the sensorimotor neocortex during visual processing, with a simultaneous alpha ERD over visual cortices, and vice versa during movement (Pfurtscheller, 1992) (see also Rougeul et al., 1979). Further, premovement alpha ERD is accentuated in elite athletes over the entire brain, suggesting that this is a functionally relevant mechanism that can be enhanced with practice (Del Percio et al., 2009).

Several studies have shown that selective somatic attention impacts movement- or sensory-induced ERD and ERS changes in sensorimotor mu-alpha and mu-beta activity (Bauer et al., 2006; Babiloni et al., 2008; Dockstader et al., 2010). However, allocation of these rhythms following an attentional cue, in anticipation of tactile sensory processing, has not been investigated.

In the present study, we used MEG imaging to investigate whether similar effects to those observed in the visual system are also present in somatosensation, using the well localized hand representation in SI as our substrate (Jones et al., 2007, 2009). We investigated whether spatial attention directed to or away from 
the hand impacted allocation of mu-alpha and mu-beta, the predictive value of these rhythms for detection, and the impact of cued attention on evoked responses. We observed an impact in each of these dimensions, indicating that the decreased expression of localized alpha oscillations could be causally beneficial to attentional regulation employed across neocortical areas.

\section{Materials and Methods}

\section{Subjects}

MEG data were collected from 12 neurologically healthy (exclusion criteria included musculoskeletal diseases, arthritis, lupus, multiple sclerosis, scleroderma, and diagnosed current psychiatric disorder), right-handed, 18- to 50 -year-old adults (mean age $=31.6$ years, $S D=7$ years, 1 male and 11 female). Subjects were medication free or on stable doses of selective serotonin reuptake inhibitor medication. The experimental protocol was approved by the Massachusetts General Hospital Internal Review Board, and each subject gave informed consent before data acquisition.

\section{Stimuli}

Subjects' hand and foot rested on solid plastic frames through which tactile stimuli were delivered. The stimulus (single cycle of a $100 \mathrm{~Hz}$ sine wave, $10 \mathrm{~ms}$ duration) was generated by fused multilayer piezoelectric benders, which provide more favorable force and higher-frequency resonance characteristics than typical ceramic wafers (Noliac) (see Jones et al., 2007). Stimuli were applied to the distal pads of the third digit of the left hand and first digit of the left foot via a Delrin contactor affixed to the piezoelectric bender $(7 \mathrm{~mm}$ diameter presented within a $1 \mathrm{~cm}$ circular rigid surround). The device was not glued to the skin. Instead, matched intensity of stimulation, relative to perceptual threshold, was maintained individually for each subject using a parameter estimation sequential testing (PEST) convergence procedure (Dai, 1995; Leek, 2001). During the cued detection runs described below, stimulus strength was maintained at $66 \%$ detection threshold with suprathreshold (100\% detected) and null stimuli randomly interleaved for $10 \%$ and $20 \%$ of the trials, respectively. The PEST procedure and the hand stimulation device and protocol were as used by Jones et al. $(2007,2009)$.

\section{Experimental procedure}

Localization runs. To aid localization of primary equivalent current dipoles (ECDs) in contralateral SI, each experiment began with presentation of suprathreshold stimuli to the left hand third digit for $3 \mathrm{~min}$ with an interstimulus interval of $3 \mathrm{~s}$ ( 60 trials per subject). Separate localization runs were also performed on the first digit of the foot, as an original goal of our study was to look at the somatotopic precision of attention allocation by comparing hand and foot activity. However, consistent dipoles could not be reconstructed from the foot localization data using the standard ECD localization techniques described below. Thus, only hand area activity is presented, as its precise position in the SI map could be confirmed.

Cued detection runs. Subjects were instructed to fixate on a cross on a projection screen. PEST procedure was used for $3 \mathrm{~min}$ at the beginning to determine subjects' initial detection thresholds. This run was followed by at least 5 cued detection runs, described in Figure 1, consisting of a $3.5 \mathrm{~s}$ trial that began with the fixation cross changing into a visual word cue on a projection screen directing the participant to attend to the "Hand" (the attend-in condition), the "Foot" (the attend-out condition), or "Either" location. The visual cue was accompanied by a $60 \mathrm{~dB}, 2 \mathrm{kHz}$ tone delivered to both ears to mask audible clicks created by the tactile stimulator and remained constant for $2.5 \mathrm{~s}$. At a randomized time between 1.1 and $2.1 \mathrm{~s}$ (fixed $100 \mathrm{~ms}$ intervals) after the visual cue, the piezoelectric stimulator delivered a brief tactile stimulus to either the finger or toe. At the end of the $2.5 \mathrm{~s}$ visual cue, and at least $400 \mathrm{~ms}$ after tactile stimulus, subjects reported detection or nondetection of the stimulus at the cued location with button presses using the second and third digits of the right hand, respectively. The next trial began $1 \mathrm{~s}$ after cessation of the visual cue. There were 120 trials per run, 40 of each attention condition, totaling at least 200 trials of 3 stimulus strengths in each condition.

MEG data acquisition and source analysis. The MEG signals were recorded using a 306-channel whole-head planar dc-SQUID Neuromag
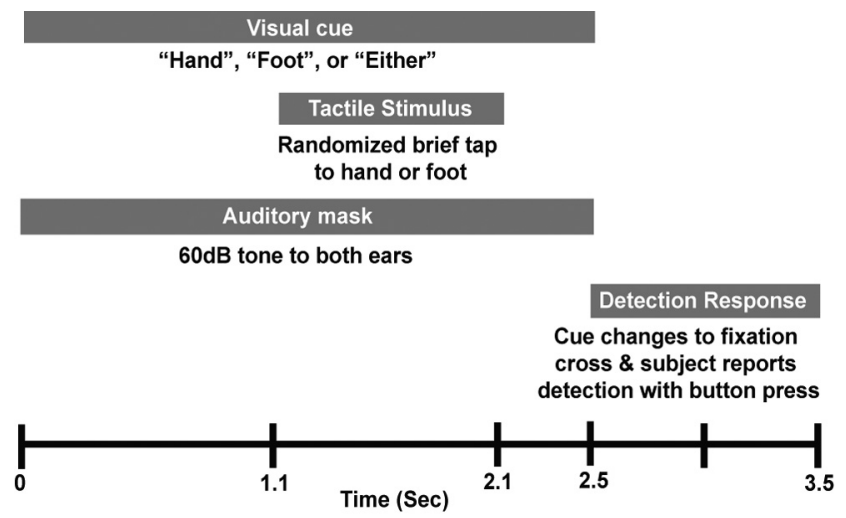

Figure 1. Experimental design for cued detection runs. See Materials and Methods.

Vectorview system. Data were acquired at $601 \mathrm{~Hz}$ and filtered from 0.1 to $200 \mathrm{~Hz}$. Four head position coils recorded head position in the Dewar for coregistration with structural MR images. Vertical and horizontal electro-oculogram (EOG) signals were recorded with electrodes placed close to the left eye. Epochs with EOG peak-to-peak amplitude exceeding $100 \mu \mathrm{V}$ were excluded from analysis.

The contribution from the left third digit representation in SI to the measured fields was estimated using a least-squares fit with a dipole forward solution calculated using a spherically symmetric conductor model of the head (Hämäläinen and Sarvas, 1989). Averaged data from the localization runs described above were used to find an ECD (ElektaNeuromag software) at the time of peak activity (mean peak activity $=$ $66.8 \mathrm{~ms}, \mathrm{SD}=6.4 \mathrm{~ms}$ ) in the mean signal from the suprathreshold stimuli (minimum $n=50$ runs per subject). The goodness of fit of this single dipole model was larger than $70 \%$ in all fit data during peak responses. Coregistration of the SI source localization with the individual's anatomical MRIs confirmed that the source emerged from the anterior band of the postcentral gyrus finger representation of area $3 \mathrm{~b}$ in SI (Moore et al., 2000) in all subjects (see Fig. 2A). All analysis considered the forward solution from this SI source.

\section{Analysis}

Time evolution of spectral power. This metric was calculated using a complex wavelet analysis, from which time-frequency representations (TFRs) of near instantaneous changes were determined. The TFRs were calculated from 1 to $40 \mathrm{~Hz}$ on the SI ECD time courses by convolving signals with a complex Morlet wavelet of the form $w\left(t, f_{0}\right)=A \exp \left(-t^{2} /\right.$ $\left.2\left[\sigma_{t}^{2}\right]\right) \exp \left(2 i \pi f_{0} t\right)$, for each frequency of interest $f_{0}$, where $\sigma_{t}=m / 2 \pi f_{0}$, and $i$ is the imaginary unit. The normalization factor was $A=$ $1 /\left(\sigma_{t} \sqrt{2 \pi}\right)$, and the constant $m$ defining the compromise between time and frequency resolution was 7 , as in the Jones et al. (2009) study. Timefrequency representations of mu-alpha and mu-beta power were calculated as the squared magnitude of the complex wavelet-transformed data averaged across the range of interest. For the postcue analysis (see Fig. $2 B)$, baseline was calculated as averaged power $[-200,0]$ ms relative to the cue-averaged across attend-in and attend-out trials separately. For the prestimulus analysis (see Fig. $2 C$ ), baseline was calculated as average power $[-500,0]$ ms relative to the stimulus.

Data were analyzed using the last 100 trials of each condition. This choice reflects preliminary analysis across multiple studies in our laboratory (S. R. Jones and C. I. Moore, unpublished data) indicating that the initial 100 trials provide less stable and consistent neurophysiological activity patterns across individuals.

Visual cue and tactile stimulus broadband evoked responses. SI evoked responses were calculated across an equal number of attend-in and attend-out trials per subject from threshold-level tactile stimulation to the finger (number of trials mean $=88$ trials; $S D=10$ ). Averages were baseline normalized by subtracting the mean over $[-100,0] \mathrm{ms}$ from the cue or stimulus, for each subject (see Fig. 3). A response artifact from 1.7 to $2.1 \mathrm{~s}$ in one subject was removed from Figure $3 A$. 
Impact of mu-alpha and mu-beta on detection probabilities. We could not use the current data to assess the impact of mu-alpha and mu-beta on detection probabilities because the subset of relevant data was a statistically small sample. The relevant data consisted of the "hit" and "miss" trials in the attend-hand condition, and there were only a small number of miss trials per subject in this subcondition (number of miss trials: mean $=11, \mathrm{SD}=6$; number of hit trials: mean $=83, \mathrm{SD}=11$ ). Therefore, we conducted analysis using a second dataset where the statistics were tractable. As in the present study, subjects detected taps applied to the third digit fingertip (of the right hand) using the same stimulator, and signals were localized to the hand dipole using identical means. Because foot trials and "either" trials were not interleaved-subjects attended to the hand throughout-we had a more extensive trial base for comparing hit and miss trials (last 100 trials analyzed). Details of data collection were described in detail in prior reports by our group (Jones et al., 2007, 2009; Ziegler et al., 2010).

Analysis methods were as in the LinkenkaerHansen et al. (2004) study. In brief, for each subject mu-alpha and mu-beta power was averaged over a $1 \mathrm{~s}$ prestimulus time window for each of the last 100 threshold-level stimulus trials, and binned into 10 power percentile bins (10 averages per bin) sorted from low to high. The probability of detection in each bin was calculated as a percentage change in hit rate (number of detected trials/total number of trials) from the mean (see Fig. 4).

Statistical analyses. Nonparametric Wilcoxon sign-rank tests were used to assess the statistical significance of differences in attend-in and attend-out conditions across subjects at every time point (see Figs. $2 B, C, 3$ ). The Wilcoxon test was preferred over ANOVA because the data were non-normally distributed (ShapiroWilks test). Linear regression on the mean percentage change in hit rate across subjects was used to assess the impact of mu-alpha and mubeta power on detection (see Fig. 4).

\section{Results}

We investigated the temporal evolution of changes in mu-alpha $(7-14 \mathrm{~Hz})$ and mu-beta $(15-29 \mathrm{~Hz})$ power in a localized dipole source in the right hand area of SI after a cue to attend to tactile finger stimulation to the contralateral left hand (attend-in condition), or to tactile toe-stimulation to the left foot (attend-out condition). Figure $2 \mathrm{~A}$ shows example localizations in two subjects in the SI hand representation in the anterior bank of the postcentral gyrus (area $3 \mathrm{~b}$ ), confirmed by proximity to the $\Omega$-shaped bend (marked in red in Fig. $2 A$, bottom panel) in the central sulcus (Moore et al., 2000). Figure $2 B$ shows the corresponding average percentage change from baseline $[n=12$ subjects (Ss)] in SI mu-alpha and mu-beta power during the time period $[-100,1100] \mathrm{ms}$ relative to the cue in attend-in and attend-out conditions. A significant difference across subjects was observed between the conditions in the mu-alpha band during the anticipatory postcue time period $[500,1100] \mathrm{ms}$ relative to the cue $(p<0.05$ marked with asterisks, Wilcoxon sign-rank
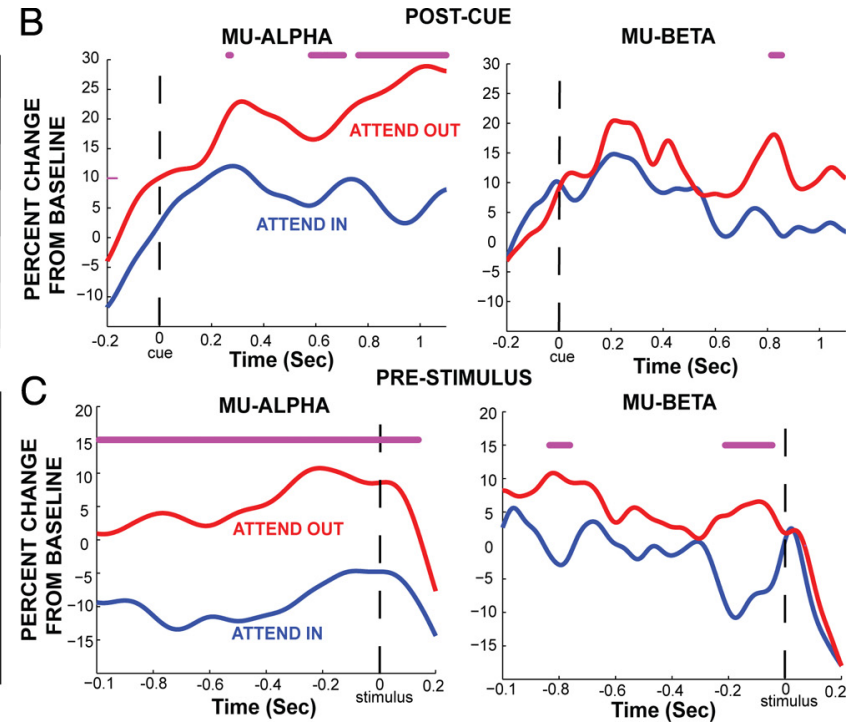

Figure 2. Impact of cued attention on SI mu-alpha and mu-beta. $\boldsymbol{A}$, Two examples of the estimated SI ECD localizations (blue (he 3 shape (marked in red bottom panel), in the anterior bank of the contralateral postcentral gyrus. $\boldsymbol{B}$, Continuous postcue temporal evolution of the hand area $S$ mu-alpha (7-14 Hz) and mu-beta (15-29 Hz) activity in attend-in and attend-out conditions (avg. $n=12$ Ss). C, Corresponding

A

Figure 3. Impact of cued attention visual cue and tactile stimulus SI ERs. A, Average hand area SI broadband ER from the visual threshold-level tactile stimulation to the hand in attend-in and attend-out conditions. Asterisks, Significant difference between conditions $(p<0.05)$. test). Significant differences in the mu-beta band were observed for a time window of $[800,850] \mathrm{ms}$.

Aligning trials to the tactile stimulus onset $([-1000,200] \mathrm{ms})$, rather than visual cue, also showed a dominant effect of cued attention on prestimulus mu-alpha activity (Fig. 2C). Significant differences between attend-in and attend-out conditions are seen in the mu-alpha across the entire prestimulus time period, and in the $[-200,0] \mathrm{ms}$ time window for mu-beta, with another period of significance around $-800 \mathrm{~ms}$.

Next, we investigated attentional modulation of the broadband SI evoked response to the visual cue and subsequent threshold-level tactile stimulus (Fig. 3). There was a rapid response in SI to the visual cue with an initial peak near $70 \mathrm{~ms}$ (labeled pcM70, for "postcue M70," for reference in Fig. 3A) that was greater in the attend-in condition. Several other time points showed a significant difference between attend-in and attend-out conditions, and the timings of these differences was consistent with previously reported modulation of EEG measured eventrelated potentials (ERPs) during attention deployment in parietal, frontal, and visual cortices (Kelly et al., 2009). Most notable are the statistically significant differences at $200 \mathrm{~ms}, 400 \mathrm{~ms}$, and 
several intervals between 500 and $900 \mathrm{~ms}$ (see magenta asterisks in Fig. 3A). The $200 \mathrm{~ms}$ difference is consistent with that seen in parietal cortices and typically referred to as an "early directing attention negativity" (EDAN), labeled pcMEDAN (for "postcue MEG EDAN") in Figure 3A. The difference near $400 \mathrm{~ms}$ is consistent with the anterior directing attention negativity (ADAN) observed in frontal cortices, and the later noncontinuous differences between 500 and $900 \mathrm{~ms}$ are in line with those seen over occipital cortex known as late directing attention positivity (LDAP), labeled pcMADAN and pcMLDAP, respectively.

Figure $3 B$ shows the SI tactile ER at $[-100,200]$ ms. Peaks in the waveform were consistent with previous reports using similar stimuli (Jones et al., 2007, 2009). Four peaks occurring at $\sim 50 \mathrm{~ms}$ (M50), $70 \mathrm{~ms}$ (M70), $100 \mathrm{~ms}$ (M100), and $135 \mathrm{~ms}$ (M135), respectively, are labeled for visualization as in the Jones et al. (2007, 2009) studies. A previous study showed that high prestimulus mu-alpha and mu-beta was correlated with an increase in the magnitude of the M50 peak and a subsequent trend toward decreased M70 and later response elements (Jones et al., 2009). Here, we found that there was also a significant difference in the magnitude of the ER near the M50 peak between the attend-in and attend-out conditions, such that the magnitude of the ER was greater in the attend-out conditions, when prestimulus mu-alpha and mu-beta were higher (Fig. $3 B$, significant time points marked with asterisks, $p<0.05$ Wilcoxon sign-rank test). There was also a significant difference in the ER near the M100 peak $(p<0.05$ Wilcoxon sign-rank).

Although a slow cue-locked fluctuation is apparent visually in the averaged cue ER in attend-in and attend-out conditions (Fig. $3 A$ ), this did not bias the averaged tactile ER (Fig. $3 B$ ) since the tactile stimulus was jittered within the $[1.1,2.1]$ s postcue time window (marked in Fig. 3A). However, it is possible this early ER was impacted by postcue attentional modulation of the mu-alpha and mu-beta activity that is not phase locked to the cue (Fig. 2) (see Discussion).

We assessed trial-by-trial impact of prestimulus mu-alpha and mu-beta in our SI signal on tactile detection probabilities, using a second dataset that used analogous MEG and tactile detection methods, but with sustained attention to the finger (Jones et al., 2007, 2009; Ziegler et al., 2010). This previously collected dataset gave greater statistical power than the current data, where the relevant hit and miss trials represented a statistically small sample (see Materials and Methods). Following prior convention (Linkenkaer-Hansen et al., 2004), on individual trials average prestimulus mu-alpha and mu-beta power was calculated (1 s before stimulus) and sorted from high to low power into 10 equally sized percentile bins. Detection probabilities in each bin were calculated as the percentage change in hit rate from the mean (see Materials and Methods). We found a linear relationship between tactile detection probabilities and mu-alpha and mu-beta power $\left(p<0.05, F\right.$ test, $R^{2}=0.65$ and $R^{2}=0.85$, respectively) such that the hit probability was greater during trials with lower prestimulus mu-alpha and mu-beta power (Fig. 4).

\section{Discussion}

Consistent with findings in visual cortices, we observed that cued attention modulates anticipatory postcue mu-alpha activity and early peaks in the broadband visual cue- and tactile stimulusinduced ERs in the SI hand representation. We found a significant difference in the postcue change from baseline of mu-alpha power between attend-in and attend-out conditions. Detection probabilities were greater during lower prestimulus mu-alpha and mu-beta power. These results are consistent with the theory
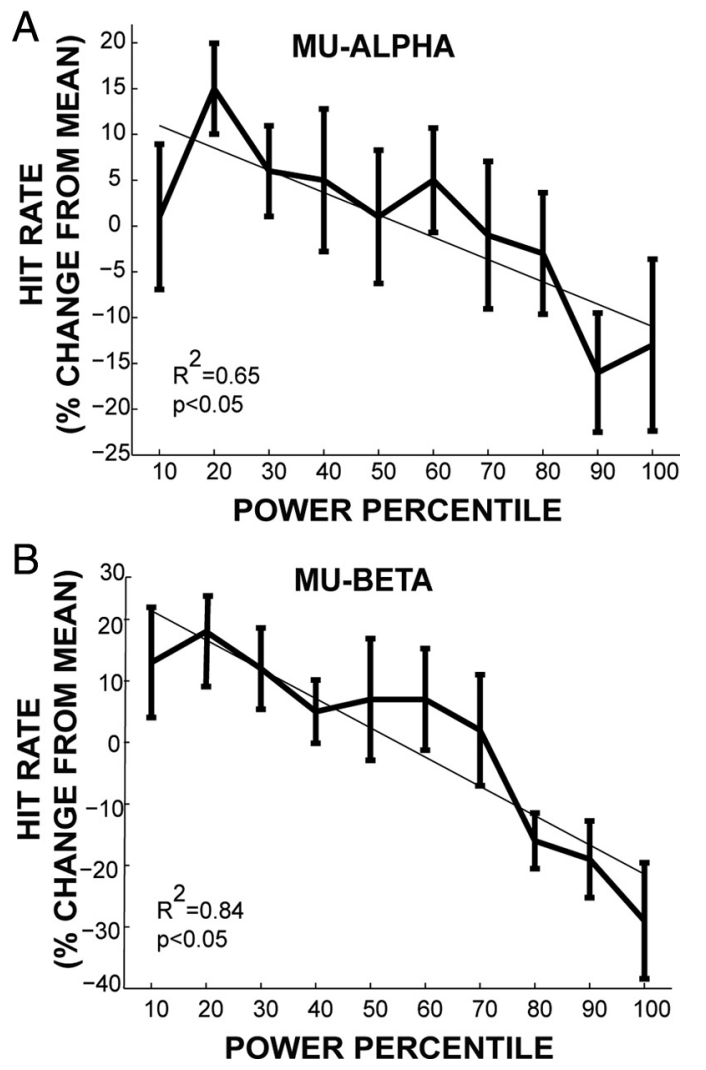

Figure 4. Impact of prestimulus mu-alpha and mu-beta power on detection probabilities. $\boldsymbol{A}$, Tactile detection probabilities, measured as percentage change in hit rate from the mean, as a function mu-alpha power sorted into 10 power percentile bins. Bold traces, Mean and SE across subjects ( $n=10 \mathrm{Ss}$ ); thin traces, linear fit from linear regression analysis $\left(R^{2}=0.65, p<0.05\right)$. $\boldsymbol{B}$, Analogous traces as a function of mu-beta power $\left(R^{2}=0.84, p<0.05\right)$. This analysis was performed on data from the Jones et al. $(2007,2009)$ studies (see Materials and Methods).

that attentionally induced focal alpha changes are an active mechanism for modulation of sensory information processing (Foxe et al., 1998; Worden et al., 2000; Kelly et al., 2006, 2009; Thut et al., 2006; Klimesch et al., 2007). Further, our results show that although attentional cuing has a weaker impact on SI mu-beta rhythms, they also predict tactile detection.

\section{Attentional modulation of low-frequency rhythms and their impact on perception}

Prior research connecting low-frequency oscillations and cued attention in somatosensory cortex in humans has focused on sensory-stimulus- and movement-induced ERD and subsequent ERS in alpha and beta frequencies (Bauer et al., 2006; Babiloni et al., 2008; Dockstader et al., 2010) (see also Pfurtscheller, 1992). These studies also investigated attentional modulation of somatosensory gamma $(35-80 \mathrm{~Hz})$ activity. Gamma was not a focus of our study because it is not robust in our spontaneous SI signal (see Jones et al., 2009).

Our finding of a linear relationship between mu-alpha and mu-beta power and tactile detection agrees with Schubert et al. (2009), who found a linear relationship between alpha and beta amplitudes in EEG electrodes over SI and tactile detection probability. These results also are consistent with the relationship between smaller alpha amplitudes and increased perception and decreased reaction times in the visual system (Thut et al., 2006; Hanslmayr et al., 2007; van Dijk et al., 2008; Kelly et al., 2009). Other studies in the somatosensory (Linkenkaer-Hansen et al., 
2004; Zhang and Ding, 2010) and visual (Rajagovindan and Ding, 2010) systems have reported an inverted-U relationship between alpha power and detection probabilities. We also found that, on trials with the lowest values of mu-alpha power, detection probability diminished compared to slightly higher values (compare 10th and 20th percentiles in Fig. 4), suggesting that a minimal baseline of alpha activity is necessary for optimal signal propagation. These results imply that the reduction of mu-alpha and mu-beta activity in SI with cued attention is an active cortical gating mechanism that increases the perceptual salience of tactile signals.

\section{Attentional modulation of visual cue and tactile stimulus SI ERs}

Studies have shown consistent effects regarding the timing and spatial specificity of distinct components of postcue EEG measured ERPs in parietal, frontal, and low-level visual areas (e.g., Kelly et al., 2009). We have shown that attentional modulation of broadband visual cue-induced ERs components exists at similar times in our high-resolution localized SI signal. In addition to changes near $200 \mathrm{~ms}$ (pcMEDAN), $400 \mathrm{~ms}$ (pcMADAN), and between 500 and 900 ms activity (pcMLDAP), we found an earlier significant difference at $\sim 70 \mathrm{~ms}$ (pcM70), such that the magnitude of the peak was greater in attend-in conditions (Fig. $3 A$ ), suggesting rapid attentional modulation in SI.

While fitting data to a single dipole is a necessary simplification in our study, the pcM70 response difference is likely restricted to activity from SI. Rapid responses $(<100 \mathrm{~ms})$ to visual stimuli have been observed previously in SI. Zhou and Fuster $(1997,2000)$ showed a change in firing rate (predominantly increases) in single units in the anterior parietal cortex of trained monkeys, including the hand representation area in SI, during a visual cue indicating a subsequent tactile choice. In this visuohaptic memory task, a change in firing rate was observed as early as $\sim 50-100 \mathrm{~ms}$ after visual cue and lasted throughout a memory retention period $(\sim 20 \mathrm{~s})$. Further, some units showed differential activity depending on haptic choice indicated by the cue-vertical or horizontal bars indicating corresponding rod with oriented ridges to pull (Zhou and Fuster, 1997, 2000).

Our findings of increased magnitude near the M50 and M100 SI tactile ER peaks (Fig. 3B) with attention are consistent with the theory that attention increases the gain of sensory-evoked responses, and are in line with previous studies that showed increased magnitudes of evoked SI activity in MEG/EEG sensor data at similar time points (Schubert et al., 2008; Dockstader et al., 2010). However, the decreased M50 peak in the attend-in condition contrasts with previous studies showing increases near $50 \mathrm{~ms}$ with attention (Iguchi et al., 2002; Schubert et al., 2008; Dockstader et al., 2010). These discrepancies are likely due to differences in experimental design and the fact that the previous studies used stimuli that were perceptually salient (electrical and Braille stimuli), in contrast to our stimulus amplitude that was maintained at a $50 \%$ perceptual threshold. The $\sim 50 \mathrm{~ms}$ differences in Iguchi et al. (2002) appeared during a two-finger discrimination task, but not during a nondiscrimination task. Schubert et al. (2008) used an oddball detection paradigm, and Dockstader et al. (2010) engaged attention by counting stimuli. Further, the relative magnitude of evoked activity in SI can vary as a function of context, with identical stimuli causing enhanced or suppressed responses dependent on the amplitude of sensory drive (Moore et al., 1999). In our study, greater prestimulus mu activity may similarly prime the enhancement of this early response component under conditions of weak sensory drive.

\section{Biophysical mechanisms of SI attentional modulation: connection to previous computational modeling predictions SI mu-alpha rhythms}

We have developed a model of SI that predicts the origin of the MEG mu rhythm and tactile ERs (Jones et al., 2007, 2009). The model predicts that the mu-alpha component of the mu rhythm is created by a $10 \mathrm{~Hz}$ lemniscal thalamic input to granular and infragranular layers, while the mu-beta component is dependent on a second $10 \mathrm{~Hz}$ input to the supragranular layers. Given our model, the observed attentional decrease of mu-alpha is predicted to occur through suppression of ongoing $10 \mathrm{~Hz}$ lemniscal thalamus activity. This is consistent with the notion proposed by Francis Crick that the inhibitory reticular thalamus controls an attentional "searchlight" in topographic sensory representations (Crick, 1984) and recent recordings by McAlonan et al. (2008) showing that attention modulates sensory-evoked thalamic responses before reaching the neocortex (see also Suffczynski et al., 2001).

\section{SI ERs}

Our previous modeling further predicts that the decreased M50 tactile stimulus peak responses with attention may arise, at least in part, from excitatory neurons that are less depolarized during low prestimulus mu and hence have a smaller immediate poststimulus (M50) response. This decreased excitatory response leads to decreased poststimulus inhibition in the network, which enables greater subsequent M70 and M100 responses (see Jones et al., 2009). Recent research suggests that alpha rhythms may be amplified by a recruitment of a class of low-threshold spiking inhibitory neurons (Fanselow et al., 2008; Vierling-Claassen et al., 2010). These predictions provide a direct neural correlate for the theory that mu-alpha exerts inhibitory control in the neocortex (Worden et al., 2000; Klimesch et al., 2007).

Further, the negative polarity of the postcue SI pcM70 suggests it was driven by excitatory input to the supragranular layers, from higher-order neocortical or nonspecific thalamic sources (Jones et al., 2007). A subsequent peak with negative polarity emerges $\sim 100$ ms later, reflective of a two-cycle cue-locked $\sim 10$ $\mathrm{Hz}$ drive, which is stronger with attention. The attentional differences between 500 and $900 \mathrm{~ms}$ (pcMLDAP) appear to be tied to the cue-locked slow fluctuation. Slow oscillations are known to modulate higher-frequency rhythms (Schroeder and Lakatos, 2009); however, an investigation of this is beyond the scope of this study.

In summary, our results support the idea that alpha deployment may be a general mechanism of cued attention for active sensory gating. Mu-beta rhythms are impacted less by attentional cuing, but are well correlated with the probability of tactile detection. This dissociation suggests that while both oscillations impact signal processing, mu-alpha deployment may be more readily recruited during attentional allocation. Future research is needed to identify whether attentional mu-alpha modulation is somatotopically precise on finer spatial scales, as has been explored in the visual system.

\section{References}

Babiloni C, Capotosto P, Brancucci A, Del Percio C, Petrini L, Buttiglione M, Cibelli G, Romani GL, Rossini PM, Arendt-Nielsen L (2008) Cortical alpha rhythms are related to the anticipation of sensorimotor interaction between painful stimuli and movements: a high-resolution EEG study. J Pain 9:902-911.

Bauer M, Oostenveld R, Peeters M, Fries P (2006) Tactile spatial attention enhances gamma-band activity in somatosensory cortex and reduces lowfrequency activity in parieto-occipital areas. J Neurosci 26:490-501. 
Crick F (1984) Function of the thalamic reticular complex: the searchlight hypothesis. Proc Natl Acad Sci U S A 81:4586-4590.

Dai H (1995) On measuring psychometric functions: a comparison of the constant-stimulus and adaptive up-down methods. J Acoust Soc Am 98:3135-3139.

Del Percio C, Babiloni C, Bertollo M, Marzano N, Iacoboni M, Infarinato F, Lizio R, Stocchi M, Robazza C, Cibelli G, Comani S, Eusebi F (2009) Visuo-attentional and sensorimotor alpha rhythms are related to visuomotor performance in athletes. Hum Brain Mapp 30:3527-3540.

Dockstader C, Cheyne D, Tannock R (2010) Cortical dynamics of selective attention to somatosensory events. Neuroimage 49:1777-1785.

Fanselow EE, Richardson KA, Connors BW (2008) Selective, state-dependent activation of somatostatin-expressing inhibitory interneurons in mouse neocortex. J Neurophysiol 100:2640-2652.

Foxe JJ, Simpson GV, Ahlfors SP (1998) Parieto-occipital approximately 10 $\mathrm{Hz}$ activity reflects anticipatory state of visual attention mechanisms. Neuroreport 9:3929-3933.

Fu KM, Foxe JJ, Murray MM, Higgins BA, Javitt DC, Schroeder CE (2001) Attention-dependent suppression of distracter visual input can be crossmodally cued as indexed by anticipatory parieto-occipital alpha-band oscillations. Brain Res Cogn Brain Res 12:145-152.

Hämäläinen MS, Sarvas J (1989) Realistic conductivity geometry model of the human head for interpretation of neuromagnetic data. IEEE Trans Biomed Eng 36:165-171.

Hanslmayr S, Aslan A, Staudigl T, Klimesch W, Herrmann CS, Bäuml KH (2007) Prestimulus oscillations predict visual perception performance between and within subjects. Neuroimage 37:1465-1473.

Iguchi Y, Hoshi Y, Tanosaki M, Taira M, Hashimoto I (2002) Selective attention regulates spatial and intensity information processing in the human primary somatosensory cortex. Neuroreport 13:2335-2339.

Jones SR, Pritchett DL, Stufflebeam SM, Hämäläinen M, Moore CI (2007) Neural correlates of tactile detection: a combined MEG and biophysically based computational modeling study. J Neurosci 27:10751-10764.

Jones SR, Pritchett DL, Sikora MA, Stufflebeam SM, Hämäläinen M, Moore CI (2009) Quantitative analysis and biophysically realistic neural modeling of the MEG mu rhythm: rhythmogenesis and modulation of sensory-evoked responses. J Neurophysiol 102:3554-3572.

Kelly SP, Lalor EC, Reilly RB, Foxe JJ (2006) Increases in alpha oscillatory power reflect an active retinotopic mechanism for distracter suppression during sustained visuospatial attention. J Neurophysiol 95:3844-3851.

Kelly SP, Gomez-Ramirez M, Foxe JJ (2009) The strength of anticipatory spatial biasing predicts target discrimination at attended locations: a highdensity EEG study. Eur J Neurosci 30:2224-2234.

Klimesch W, Sauseng P, Hanslmayr S (2007) EEG alpha oscillations: the inhibition-timing hypothesis. Brain Res Rev 53:63-88.

Leek MR (2001) Adaptive procedures in psychophysical research. Percept Psychophys 63:1279-1292.

Linkenkaer-Hansen K, Nikulin VV, Palva S, Ilmoniemi RJ, Palva JM (2004) Prestimulus oscillations enhance psychophysical performance in humans. J Neurosci 24:10186-10190.

McAlonan K, Cavanaugh J, Wurtz RH (2008) Guarding the gateway to cortex with attention in visual thalamus. Nature 456:391-394.

Moore CI, Nelson SB, Sur M (1999) Dynamics of neuronal processing in rat somatosensory cortex. Trends Neurosci 22:513-520.
Moore CI, Stern CE, Corkin S, Fischl B, Gray AC, Rosen BR, Dale AM (2000) Segregation of somatosensory activation in the human rolandic cortex using fMRI. J Neurophysiol 84:558 -569.

Pfurtscheller G (1992) Event-related synchronization (ERS): an electrophysiological correlate of cortical areas at rest. Electroencephalogr Clin Neurophysiol 83:62-69.

Rajagovindan R, Ding M (2010) From prestimulus alpha oscillation to visual-evoked response: an inverted-U function and its attentional modulation. J Cogn Neurosci. Advance online publication. Retrieved September 24, 2010. doi:10.1162/jocn.2010.21478.

Rougeul A, Bouyer JJ, Dedet L, Debray O (1979) Fast somato-parietal rhythms during combined focal attention and immobility in baboon and squirrel monkey. Electroencephalogr Clin Neurophysiol 46:310-319.

Schroeder CE, Lakatos P (2009) Low-frequency neuronal oscillations as instruments of sensory selection. Trends Neurosci 32:9-18.

Schubert R, Ritter P, Wüstenberg T, Preuschhof C, Curio G, Sommer W, Villringer A (2008) Spatial attention related SEP amplitude modulations covary with BOLD signal in S1-a simultaneous EEG-fMRI study. Cereb Cortex 18:2686-2700.

Schubert R, Haufe S, Blankenburg F, Villringer A, Curio G (2009) Now you'll feel it, now you won't: EEG rhythms predict the effectiveness of perceptual masking. J Cogn Neurosci 21:2407-2419.

Suffczynski P, Kalitzin S, Pfurtscheller G, Lopes da Silva FH (2001) Computational model of thalamo-cortical networks: dynamical control of alpha rhythms in relation to focal attention. Int J Psychophysiol 43:25-40.

Thut G, Nietzel A, Brandt SA, Pascual-Leone A (2006) Alpha-band electroencephalographic activity over occipital cortex indexes visuospatial attention bias and predicts visual target detection. J Neurosci 26:9494-9502.

Tiihonen J, Kajola M, Hari R (1989) Magnetic mu rhythm in man. Neuroscience 32:793-800.

van Dijk H, Schoffelen JM, Oostenveld R, Jensen O (2008) Prestimulus oscillatory activity in the alpha band predicts visual discrimination ability. J Neurosci 28:1816-1823.

Vierling-Claassen D, Cardin J, Moore C, Jones SR (2010) Computational modeling of distinct neocortical oscillations driven by cell-type selective optogenetic drive: separable resonant circuits controlled by lowthreshold spiking and fast-spiking interneurons. Paper presented at Neuroscience 2010, Program No. 645.16, San Diego, CA, Nov. 13-17.

Worden MS, Foxe JJ, Wang N, Simpson GV (2000) Anticipatory biasing of visuospatial attention indexed by retinotopically specific alpha-band electroencephalography increases over occipital cortex. J Neurosci 20:RC63.

Zhang Y, Ding M (2010) Detection of a weak somatosensory stimulus: role of the prestimulus mu rhythm and its top-down modulation. J Cogn Neurosci 22:307-322.

Zhou YD, Fuster JM (1997) Neuronal activity of somatosensory cortex in a cross-modal (visuo-haptic) memory task. Exp Brain Res 116:551-555.

Zhou YD, Fuster JM (2000) Visuo-tactile cross-modal associations in cortical somatosensory cells. Proc Natl Acad Sci U S A 97:9777-9782.

Ziegler DA, Pritchett DL, Hosseini-Varnamkhasti P, Corkin S, Hämäläinen M, Moore CI, Jones SR (2010) Transformations in oscillatory activity and evoked responses in primary somatosensory cortex in middle age: a combined computational neural modeling and MEG study. Neuroimage 52:897-912. 